\title{
Association Between Intensive Care Unit Transfer Delay and Hospital Mortality: A Multicenter Investigation
}

\author{
Matthew M. Churpek, MD, MPH, PhD ${ }^{1 \star}$, Blair Wendlandt, MD¹, Frank J. Zadravecz, MPH${ }^{1}$, Richa Adhikari, MPH ${ }^{1}$, \\ Christopher Winslow, MD², Dana P. Edelson, MD, MS ${ }^{1}$
}

${ }^{1}$ Department of Medicine, University of Chicago, Chicago, Illinois; '2Department of Medicine, NorthShore University HealthSystem, Evanston, Illinois.

BACKGROUND: Previous research investigating the impact of delayed intensive care unit (ICU) transfer on outcomes has utilized subjective criteria for defining critical illness.

OBJECTIVE: To investigate the impact of delayed ICU transfer using the electronic Cardiac Arrest Risk Triage (eCART) score, a previously published early warning score, as an objective marker of critical illness.

DESIGN: Observational cohort study.

SETTING: Medical-surgical wards at 5 hospitals between November 2008 and January 2013.

PATIENTS: Ward patients.

INTERVENTION: None.

MEASUREMENTS: eCART scores were calculated for all patients. The threshold with a specificity of $95 \%$ for ICU transfer (eCART $\geq 60$ ) denoted critical illness. A logistic regression model adjusting for age, sex, and surgical status was used to calculate the association between time to ICU transfer from first critical eCART value and in-hospital mortality.

RESULTS: A total of 3789 patients met the critical eCART threshold before ICU transfer, and the median time to ICU transfer was 5.4 hours. Delayed transfer ( $>6$ hours) occurred in $46 \%$ of patients $(n=1734)$ and was associated with increased mortality compared to patients transferred early (33.2\% vs $24.5 \%, P<0.001)$. Each 1 -hour increase in delay was associated with an adjusted $3 \%$ increase in odds of mortality $(P<0.001)$. In patients who survived to discharge, delayed transfer was associated with longer hospital length of stay (median 13 vs 11 days, $P<0.001$ ).

CONCLUSIONS: Delayed ICU transfer is associated with increased hospital length of stay and mortality. Use of an evidence-based early warning score, such as eCART, could lead to timely ICU transfer and reduced preventable death. Journal of Hospital Medicine 2016;11:757-762. (c) 2016 Society of Hospital Medicine
Patients on hospital wards may become critically ill due to worsening of the underlying condition that was the cause of their admission or acquisition of a new hospital-acquired illness. Once physiologic deterioration occurs, some patients are evaluated and quickly transferred to the intensive care unit (ICU), whereas others are left on the wards until further deterioration occurs. Because many critical illness syndromes benefit from early intervention, such as sepsis and respiratory failure, early transfer to the ICU for treatment may improve patient outcomes, and conversely, delays in ICU transfer may lead to increased mortality and length of stay (LOS) in critically ill ward patients. ${ }^{1,2}$ However, the timeliness of that transfer is dependent on numerous changing variables, such as ICU bed availability, clinician identification of the deterioration, and clinical judgment regarding the appropriate

*Address for correspondence and reprint requests: Matthew $\mathrm{M}$. Churpek, MD, University of Chicago Medical Center, Section of Pulmonary and Critical Care Medicine, 5841 South Maryland Avenue, MC 6076, Chicago, IL 60637; Telephone: 773-702-1092; Fax: 773-702-6500; E-mail: matthew.churpek@uchospitals.edu

Additional Supporting Information may be found in the online version of this article.

Received: January 26, 2016; Revised: May 20, 2016; Accepted: May 24, 2016

2016 Society of Hospital Medicine DOI 10.1002/jhm.2630

Published online in Wiley Online Library (Wileyonlinelibrary.com). transfer thresholds. ${ }^{2-7}$ As a result, there is a large degree of heterogeneity in the severity of illness of patients at the time of ICU transfer and in patient outcomes. ${ }^{6,8}$

Previous studies investigating the association between delayed ICU transfer and patient outcomes have typically utilized the time of consultation by the ICU team to denote the onset of critical illness. $5,6,9,10$ However, the decision to transfer a patient to the ICU is often subjective, and previous studies have found an alarmingly high rate of errors in diagnosis and management of critically ill ward patients, including the failure to call for help. ${ }^{2,11}$ Therefore, a more objective tool for quantifying critical illness is necessary for determining the onset of critical illness and quantifying the association of transfer delay with patient outcomes.

Early warning scores, which are designed to detect critical illness on the wards, represent objective measures of critical illness that can be easily calculated in ward patients. ${ }^{12}$ The aim of this study was to utilize the electronic Cardiac Arrest Risk Triage (eCART) score, a previously published, statistically derived early warning score that utilizes demographic, vital sign, and laboratory data, as an objective measure of critical illness to estimate the effect of delayed ICU transfer on patient outcomes in a large, multicenter database. ${ }^{13}$ We chose 6 hours as the cutoff for delay 
in this study a priori because it is a threshold noted to be an important time period in critical illness syndromes, such as sepsis. ${ }^{14,15}$

\section{METHODS}

All patients admitted to the medical-surgical wards at 5 hospitals between November 2008 and January 2013 were eligible for inclusion in this observational cohort study. Further details of the hospital populations have been previously described. ${ }^{13} \mathrm{~A}$ waiver of consent was granted by NorthShore University HealthSystem (IRB \#EH11-258) and the University of Chicago Institutional Review Board (IRB \#16995A) based on general impracticability and minimal harm. Collection of patient information was designed to comply with the Health Insurance Portability and Accountability Act of 1996 (HIPAA) regulations.

\section{Defining the Onset of Critical Illness}

The eCART score, a statistically derived early warning score that is calculated based on patient demographic, vital sign, and laboratory data, was used as an objective measure of critical illness. ${ }^{13}$ Score calculation was performed utilizing demographic information from administrative databases and time- and locationstamped vital signs and laboratory results from data warehouses at the respective institutions. In this study, a score was calculated for each time-stamped point in the entire dataset. Of note, eCART was not used in this population for patient care as this was a retrospective observational study. An eCART score at the 95\% specificity cutoff for ICU transfer from the entire dataset defined a ward patient as critically ill, a definition created a priori and before any data analysis was performed.

\section{Defining ICU Transfer Delay and Study Outcomes}

The period of time from when a patient first reached this predefined eCART score to ICU transfer was calculated for each patient, up to a maximum of 24 hours. Transfer to the ICU greater than 6 hours after reaching the critical eCART score was defined a priori as a delayed transfer to allow comparisons between patients with nondelayed and delayed transfer. A patient who suffered a ward cardiac arrest with attempted resuscitation was counted as an ICU transfer at the time of arrest. If a patient experienced more than 1 ICU transfer during the admission, then only the first ward to ICU transfer was used. The primary outcome of the study was in-hospital mortality, and secondary outcomes were ICU mortality and hospital LOS.

\section{Statistical Analysis}

Patient characteristics were compared between patients who experienced delayed and nondelayed ICU transfers using $t$ tests, Wilcoxon rank sums, and $\chi^{2}$ tests, as appropriate. The association between length of transfer delay and in-hospital mortality was calculated using logistic regression, with adjustment for age, sex, and surgical status. In a post hoc sensitivity analysis, additional adjustments were made using each patient's first eCART score on the ward, the individual vital signs and laboratory variables from eCART, and whether the ICU transfer was due to a cardiac arrest on the wards. In addition, an interaction term between time to transfer and the initial eCART on the ward was added to determine if the association between delay and mortality varied by baseline severity. The change in eCART score over time was plotted from 12 hours before the time of first reaching the critical value until ICU transfer for those in the delayed and nondelayed groups using restricted cubic splines to compare the trajectories of severity of illness between these 2 groups. In addition, a linear regression model was fit to investigate the association between the eCART slope in the 8 hours prior to the critical eCART value until ICU transfer and the timing of ICU transfer delay. Statistical analyses were performed using Stata version 12.1 (StataCorp, College Station, TX), and all tests of significance used a 2-sided $P<0.05$.

\section{RESULTS}

A total of 269,999 admissions had documented vital signs on the hospital wards during the study period, including 11,995 patients who were either transferred from the wards to the ICU $(n=11,636)$ or who suffered a cardiac arrest on the wards $(\mathrm{n}=359)$ during their initial ward stay. Of these patients, 3789 reached an eCART score at the $95 \%$ specificity cutoff (critical eCART score of 60) within 24 hours of transfer. The median time from first critical eCART value to ICU transfer was 5.4 hours (interquartile range (IQR), 214 hours; mean, 8 hours). Compared to patients without delayed ICU transfer, those with delayed transfer were slightly older (median age, 73 [IQR, 60-83] years vs 71 [IQR, 58-82] years; $P=0.002$ ), whereas all other characteristics were similar (Table 1). Table 2 shows comparisons of vital sign and laboratory results for delayed and nondelayed transfers at the time of ICU transfer. As shown, patients with delayed transfer had lower median respiratory rate, blood pressure, heart rate, and hemoglobin, but higher median white blood cell count and creatinine.

Delayed transfer occurred in $46 \%$ of patients $(\mathrm{n}=1734)$ and was associated with increased inhospital mortality $(33.2 \%$ vs $24.5 \%, P<0.001)$. This relationship was linear, with each 1 -hour increase in transfer delay associated with a $3 \%$ increase in the odds of in-hospital death $(P<0.001)$ (Figure 1). The association between length of transfer delay and hospital mortality remained unchanged after controlling for age, sex, surgical status, initial eCART score on the wards, vital signs, laboratory values, and whether the ICU transfer was due to a cardiac arrest $(3 \%$ increase per hour, $P<0.001)$. This association did not 
TABLE 1. Comparisons of Patient Characteristics Among All ICU Transfer Patients and Nondelayed (Within Six Hours) and Delayed Transfers Who Reached the Critical CART Score

\begin{tabular}{lccc}
\hline Characteristic & $\begin{array}{c}\text { Transferred } \\
\text { Within } 6 \text { Hours, } \\
\mathrm{n}=2,055\end{array}$ & $\begin{array}{c}\text { Transfer } \\
\text { Delayed, } \\
\mathrm{n}=1,734\end{array}$ & P Value \\
\hline Age, median (IQR), $\mathrm{y}$ & $71(58-82)$ & $73(60-83)$ & 0.002 \\
Female sex, $\mathrm{n}(\%)$ & $1,018(49.5)$ & $847(48.8)$ & 0.67 \\
Race, $\mathrm{n}(\%)$ & & & 0.72 \\
$\quad$ Black & $467(22.7)$ & $374(21.6)$ & \\
$\quad$ White & $1,141(55.5)$ & $971(56.0)$ & \\
$\quad$ Other/unknown & $447(21.8)$ & $389(22.4)$ & \\
Surgical patient, $\mathrm{n}(\%)$ & $572(27.8)$ & $438(25.2)$ & 0.07 \\
Hospital LOS prior to first critical eCART, & $1.5(0.3-3.7)$ & $1.6(0.4-3.9)$ & 0.04 \\
$\quad$ median (lQR), d & & & \\
Total hospital LOS, median (IQR), d* & $11(7-19)$ & $13(8-21)$ & $<0.001$ \\
Died during admission, $\mathrm{n}(\%)$ & $503(24.5)$ & $576(33.2)$ & $<0.001$ \\
\hline
\end{tabular}

NOTE: Data shown are mean (standard deviation) unless otherwise noted; $n$ refers to the number of patients in each group. Abbreviations: eCART, electronic Cardiac Arrest Risk Triage; ICU, intensive care unit; IQR, interquartile range; LOS, length of stay. ${ }^{*}$ For patients who survived to hospital discharge

vary based on the initial eCART score on the wards $(P=0.71$ for interaction). Additionally, despite having similar median hospital lengths of stay prior to first

\begin{tabular}{|c|c|c|c|}
\hline & $\begin{array}{c}\text { Transferred } \\
\text { Within } 6 \text { Hours, } \\
n=2,055\end{array}$ & $\begin{array}{l}\text { Transfer } \\
\text { Delayed, } \\
n=1,734\end{array}$ & $P$ Value \\
\hline Respiratory rate, breaths/min & $23(18-30)$ & $22(18-28)$ & $<0.001$ \\
\hline Systolic blood pressure, $\mathrm{mm} \mathrm{Hg}$ & $111(92-134)$ & $109(92-128)$ & 0.002 \\
\hline Diastolic blood pressure, mm Hg & $61(50-75)$ & $59(49-71)$ & $<0.001$ \\
\hline Heart rate, beats/min & $106(88-124)$ & $101(85-117)$ & $<0.001$ \\
\hline Oxygen saturation, median (IQR), \% & $97(94-99)$ & $97(95-99)$ & 0.15 \\
\hline Temperature, ${ }^{\circ} \mathrm{F}$ & $98.0(97.2-99.1)$ & $98.0(97.1-99.0)$ & 0.001 \\
\hline $\begin{array}{c}\text { Alert mental status, number } \\
\text { of observations (\%) }\end{array}$ & $1,749(85 \%)$ & $1,431(83 \%)$ & $<0.001$ \\
\hline eCART score at time of ICU transfer & 61 (26-122) & $48(21-121)$ & 0.914 \\
\hline WBC & $10.3(7.5-14.5)$ & $11.7(8.1-17.0)$ & $<0.001$ \\
\hline Hemoglobin & $10.7(9.3-12.0)$ & $10.3(9.1-11.6)$ & $<0.001$ \\
\hline Platelet & $215(137-275)$ & $195(120-269)$ & 0.017 \\
\hline Sodium & $137(134-140)$ & $137(134-141)$ & 0.70 \\
\hline K+ & $4.1(3.8-4.6)$ & $4.2(3.8-4.7)$ & 0.006 \\
\hline Anion Gap & $10(8-13)$ & $10(8-14)$ & $<0.001$ \\
\hline $\mathrm{CO}_{2}$ & $24(20-26)$ & $23(18-26)$ & $<0.001$ \\
\hline BUN & $24(16-40)$ & $32(18-53)$ & $<0.001$ \\
\hline $\mathrm{Cr}$ & $1.2(0.9-2.0)$ & $1.5(1.0-2.7)$ & $<0.001$ \\
\hline GFR & $70(70-70)$ & $70(51-70)$ & $<0.001$ \\
\hline Glucose & $123(106-161)$ & $129(105-164)$ & 0.48 \\
\hline Calcium & $8.5(7.9-8.8)$ & $8.2(7.7-8.7)$ & $<0.001$ \\
\hline SGOT & $26(26-35)$ & $26(26-44)$ & 0.001 \\
\hline SGPT & $21(21-27)$ & $21(20-33)$ & 0.002 \\
\hline Total bilirubin & $0.7(0.7-1.0)$ & $0.7(0.7-1.3)$ & $<0.001$ \\
\hline Alk phos & $80(80-96)$ & 80 (79-111) & 0.175 \\
\hline Albumin & $3.0(2.7-3.0)$ & $3.0(2.4-3.0)$ & $<0.001$ \\
\hline
\end{tabular}

NOTE: Abbreviations: Alk phos, alkaline phosphatase; BUN, blood urea nitrogen; $\mathrm{Cr}$, creatinine; eCART, electronic Cardiac Arrest Risk Triage; GFR, glomerular filtration rate; ICU, intensive care unit; IQR, interquartile range; $\mathrm{K}+$, potassium; $\mathrm{SGOT}$, serum glutamic-oxaloacetic transaminase; $\mathrm{SGPT}$, serum glutamic pyruvic transaminase; WBC, white blood cells.

*All data are median (IQR) unless otherwise noted.

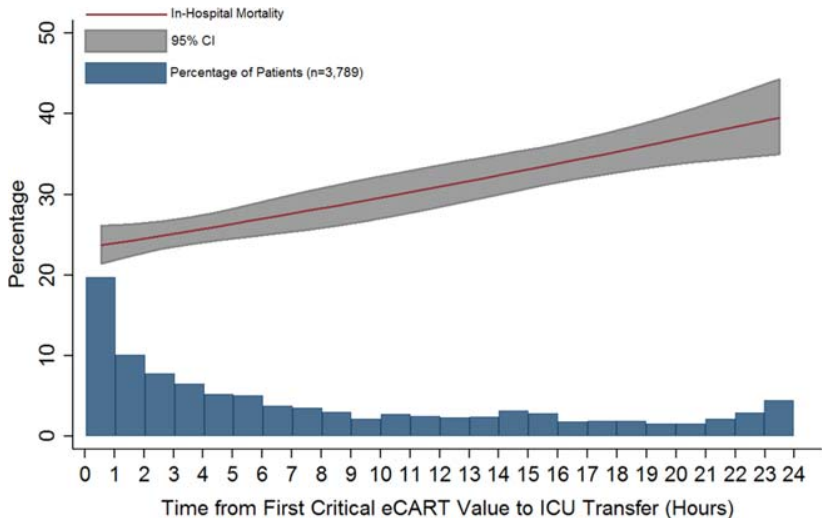

FIG. 1. Association between length of intensive care unit (ICU) transfer delay and hospital mortality. Abbreviations: $\mathrm{Cl}$, confidence interval; eCART, electronic Cardiac Arrest Risk Triage.

critical eCART score (1.6 vs 1.5 days, $P=0.04$ ), patients experiencing delayed ICU transfer who survived to discharge had a longer median hospital LOS by 2 days compared to those with nondelayed transfer who survived to discharge (median LOS, 13 (8-21) days vs $11(7-19)$ days, $P=0.01)$. The change in eCART score over time in the 12 hours before first reaching the critical eCART score until ICU transfer is shown in Figure 2 for patients with delayed and nondelayed transfer. As shown, patients transferred within 6 hours had a more rapid rise in eCART score prior to ICU transfer compared to those with a delayed transfer. This difference in trajectories between delayed and nondelayed patients was similar in patients with low $(<13)$, intermediate $(13-59)$, and high $(\geq 60)$ initial eCART scores on the wards. A regression model investigating the association between eCART slope prior to ICU transfer and time to ICU transfer demonstrated that a steeper slope was significantly associated with a decreased time to ICU transfer $(P<0.01)$.

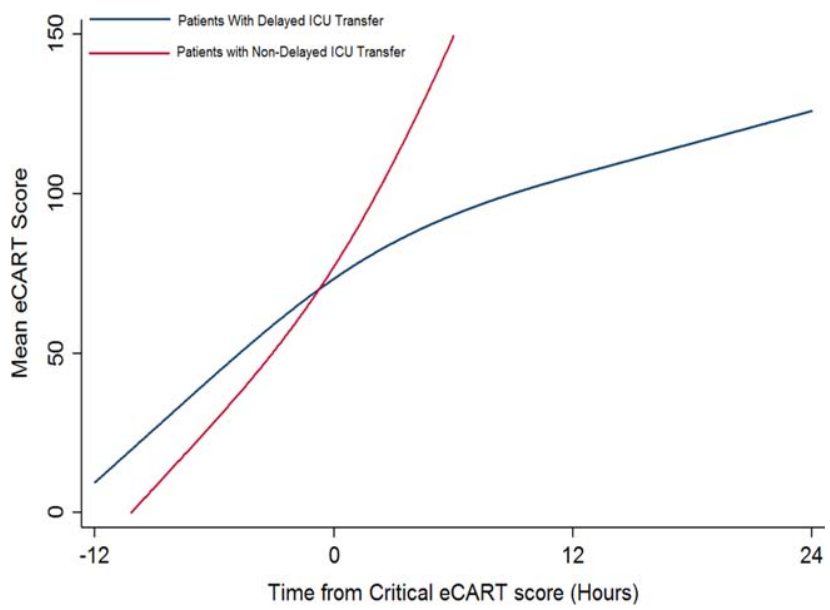

FIG. 2. Change in electronic Cardiac Arrest Risk Triage (eCART) score over time for the 12 hours prior to reaching the critical eCART value until intensive care unit (ICU) transfer for patients with delayed versus nondelayed ICU transfer. Time 0 denotes first critical eCART value. 


\section{DISCUSSION}

We found that a delay in transfer to the ICU after reaching a predefined objective threshold of critical illness was associated with a significant increase in hospital mortality and hospital LOS. We also discovered a significant association between critical illness trajectory and delays in transfer, suggesting that caregivers may not recognize more subtle trends in critical illness. This work highlights the importance of timely transfer to the ICU for critically ill ward patients, which can be affected by several factors such as ICU bed availability and caregiver recognition and triage decisions. Our findings have significant implications for patient safety on the wards and provide further evidence for implementing early warning scores into practice to aid with clinical decision making.

Our findings of increased mortality with delayed ICU transfer are consistent with previous studies. ${ }^{1,5,9}$ For example, Young et al. compared ICU mortality between delayed and nondelayed transfers in 91 consecutive patients with noncardiac diagnoses at a community hospital. ${ }^{1}$ They also used predefined criteria for critical illness, and found that delayed transfers had a higher ICU mortality than nondelayed patients $(41 \%$ vs $11 \%)$. However, their criteria for critical illness only had a specificity of $13 \%$ for predicting ICU transfer, compared to $95 \%$ in our study, suggesting that our threshold is more consistent with critical illness. Another study, by Cardoso and colleagues, investigated the impact of delayed ICU admission due to bed shortages on ICU mortality in 401 patients at a university hospital. ${ }^{9}$ Of those patients deemed appropriate for transfer to the ICU but who had to wait for a bed to become available, the median wait time for a bed was 18 hours. They found that each hour of waiting was associated with a $1.5 \%$ increase in ICU death. A similar study by Robert and colleagues investigated the impact of delayed or refused ICU admission due to a lack of bed availability. ${ }^{5}$ Patients deemed too sick (or too well) to benefit from ICU transfer were excluded. Twenty-eight-day and 60day mortality were higher in the admitted group compared to those not admitted, although this finding was not statistically significant. In addition, patients later admitted to the ICU once a bed became available (median wait time, 6 hours; $\mathrm{n}=89$ ) had higher 28-day mortality than those admitted immediately (adjusted odds ratio, 1.78; $P=0.05$ ). Several other studies have investigated the impact of ICU refusal for reasons that included bed shortages, and found increased mortality in those not admitted to the ICU. ${ }^{16,17}$ However, many of these studies included patients deemed too sick or too well to be transferred to the ICU in the group of nonadmitted patients. Our study adds to this literature by utilizing a highly specific objective measure of critical illness and by including all patients on the wards who reached this threshold, rather than only those for whom a consult was requested.
There are several potential explanations for our finding of increased mortality with delayed ICU transfer. First, those with delayed transfer might be different in some way from those transferred immediately. For example, we found that those with delayed transfer were older. The finding that increasing age is associated with a delay in ICU transfer is interesting, and may reflect physiologic differences in older patients compared to younger ones. For example, older patients have a lower maximum heart rate and thus may not develop the same level of vital sign abnormalities that younger patients do, causing them to be inappropriately left on the wards for too long. ${ }^{18} \mathrm{In}$ addition, patients with delayed transfer had more deranged renal function and lower blood pressure. It is unknown whether these organ dysfunctions would have been prevented by earlier transfer and to what degree they were related to chronic conditions. However, delayed transfer was still associated with increased mortality even after controlling for age, vital sign and laboratory values, and eCART on ward admission. It may also be possible that patients with delayed transfer received early and appropriate treatment on the wards but failed to improve and thus required ICU transfer. We did not have access to orders in this large database, so this theory will need to be investigated in future work. Finally, the most likely explanation for our findings is that earlier identification and treatment improves outcomes of critically ill patients on the wards, which is consistent with the findings of previous studies. ${ }^{1,5,9,10}$ Our study demonstrates that early identification of critical illness is crucial, and that delayed treatment can rapidly lead to increased mortality and LOS.

Our comparison of eCART score trajectory showed that patients transferred within 6 hours of onset of critical illness had a more rapid rise in eCART score over the preceding time period, whereas patients who experienced transfer delay showed a slower increase in eCART score. One explanation for this finding is that patients who decompensate more rapidly are in turn more readily recognizable to providers, whereas patients who experience a more insidious clinical deterioration are recognized later in the process, which then leads to a delay in escalation of care. This hypothesis underlines the importance of utilizing an objective marker of illness that is calculated longitudinally and in real time, as opposed to relying upon provider recognition alone. In fact, we have recently demonstrated that eCART is more accurate and identifies patients earlier than standard rapid response team activation. ${ }^{19}$

There are several important implications of our findings. First, it highlights the potential impact that early warning scores, particular those that are evidence based, can have on the outcomes of hospitalized patients. Second, it suggests that it is important to include age in early warning scores. Previous studies 
have been mixed as to whether the inclusion of age improves detection of outcomes on the wards, although the method of inclusion of age has been variable in terms of its weighting. ${ }^{20-22}$ Our study found that older patients were more likely to be left on the wards longer prior to ICU transfer after becoming critically ill. By incorporating age into early warning scores, both accuracy and early recognition of critical illness may be improved. Finally, our finding that the trends of the eCART score differed among patients who were immediately transferred to the ICU, and who had a delay in their transfer, suggests that adding vital sign trends to early warning scores may further improve their accuracy and ability to serve as clinical decision support tools.

Our study is unique in that we used an objective measure of critical illness and then examined outcomes after patients reached this threshold on the wards. This overcomes the subjectivity of using evaluation by the ICU team or rapid response team as the starting point, as previous studies have shown a failure to call for help when patients become critically ill on the wards. ${ }^{2,11,23}$ By using the eCART score, which contains commonly collected electronic health record data and can be calculated electronically in real time, we were able to calculate the score for patients on the wards and in the ICU. This allowed us to examine trends in the eCART score over time to find clues as to why some patients are transferred late to the ICU and why these late transfers have worse outcomes than those transferred earlier. Another strength is the large multicenter database used for the analysis, which included an urban tertiary care hospital, suburban teaching hospitals, and a community nonteaching hospital.

Our study has several limitations. First, we utilized just 1 of many potential measures of critical illness and a cutoff that only included one-third of patients ultimately transferred to the ICU. However, by using the eCART score, we were able to track a patient's physiologic status over time and remove the variability that comes with using subjective definitions of critical illness. Furthermore, we utilized a high-specificity cutoff for eCART to ensure that transferred patients had significantly deranged physiology and to avoid including planned transfers to the ICU. It is likely that some patients who were critically ill with less deranged physiology that would have benefitted from earlier transfer were excluded from the study. Second, we were unable to determine the cause of physiologic deterioration for patients in our study due to the large number of included patients. In addition, we did not have code status, comorbidities, or reason for ICU admission available in the dataset. It is likely that the impact of delayed transfer varies by the indication for ICU admission and chronic disease burden. It is also possible that controlling for these unmeasured factors could negate the beneficial association seen for earlier
ICU admission. However, our finding of such a strong relationship between time to transfer and mortality after controlling for several important variables suggests that early recognition of critical illness is beneficial to many patients on the wards. Third, due to its observational nature, our study cannot estimate the true impact of timely ICU transfer on critically ill ward patient outcomes. Future clinical trials will be needed to determine the impact of electronic early warning scores on patient outcomes.

In conclusion, delayed ICU transfer is associated with significantly increased hospital LOS and mortality. This association highlights the need for ongoing work toward both the implementation of an evidencebased risk stratification tool as well as development of effective critical care outreach resources for patients decompensating on the wards. Real-time use of a validated early warning score, such as eCART, could potentially lead to more timely ICU transfer for critically ill patients and reduced rates of preventable inhospital death.

\section{Acknowledgements}

The authors thank Timothy Holper, Justin Lakeman, and Contessa Hsu for assistance with data extraction and technical support; Poome Chamnankit, MS, CNP, Kelly Bhatia, MSN, ACNP, and Audrey Seitman, MSN, ACNP for performing manual chart review of cardiac arrest patients; and Nicole Twu for administrative support.

Disclosures: This research was funded in part by an institutional Clinical and Translational Science Award grant (UL1 RR024999, PI: Dr. Julian Solway). Dr. Churpek is supported by a career development award from the National Heart, Lung, and Blood Institute (K08 HL121080). Drs. Churpek and Edelson have a patent pending (ARCD. P0535US.P2) for risk stratification algorithms for hospitalized patients. In addition, Dr. Edelson has received research support from Philips Healthcare (Andover, MA), the American Heart Association (Dallas, TX), and Laerdal Medical (Stavanger, Norway). She has ownership interest in Quant HC (Chicago, IL), which is developing products for risk stratification of hospitalized patients. Drs. Churpek and Wendlandt had full access to all the data in the study and take responsibility for the integrity of the data and the accuracy of the data analysis. Preliminary versions of these data were presented at the 2015 meeting of the Society of Hospital Medicine (March 31, 2015, National Harbor, MD).

\section{References}

1. Young MP, Gooder VJ, McBride K, James B, Fisher ES. Inpatient transfers to the intensive care unit: delays are associated with increased mortality and morbidity. J Gen Intern Med. 2003;18(2):7783.

2. McQuillan P, Pilkington S, Allan A, et al. Confidential inquiry into quality of care before admission to intensive care. BMJ. 1998; 316(7148):1853-1858.

3. Town JA, Churpek MM, Yuen TC, Huber MT, Kress JP, Edelson DP. Relationship between ICU bed availability, ICU readmission, and cardiac arrest in the general wards. Crit Care Med. 2014;42(9):20372041.

4. Simchen E, Sprung CL, Galai N, et al. Survival of critically ill patients hospitalized in and out of intensive care units under paucity of intensive care unit beds. Crit Care Med. 2004;32(8):1654-1661.

5. Robert R, Reignier J, Tournoux-Facon C, et al. Refusal of intensive care unit admission due to a full unit: impact on mortality. Am J Respir Crit Care Med. 2012;185(10):1081-1087.

6. Sprung CL, Geber D, Eidelman LA, et al. Evaluation of triage decisions for intensive care admission. Crit Care Med. 1999;27(6):10731079 .

7. Garrouste-Orgeas M, Montuclard L, Timsit JF, et al. Predictors of intensive care unit refusal in French intensive care units: a multiplecenter study. Crit Care Med. 2005;33(4):750-755.

8. Zimmerman JE, Kramer AA, McNair DS, Malila FM. Acute Physiology and Chronic Health Evaluation (APACHE) IV: hospital mortality assessment for today's critically ill patients. Crit Care Med. 2006; 34(5):1297-1310. 
9. Cardoso LT, Grion CM, Matsuo T, et al. Impact of delayed admission to intensive care units on mortality of critically ill patients: a cohort study. Crit Care. 2011;15(1):R28.

10. Iapichino G, Corbella D, Minelli C, et al. Reasons for refusal of admission to intensive care and impact on mortality. Intensive Care Med. 2010;36(10):1772-1779.

11. Hodgetts TJ, Kenward G, Vlackonikolis I, et al. Incidence, location and reasons for avoidable in-hospital cardiac arrest in a district general hospital. Resuscitation. 2002;54(2):115-123.

12. Churpek MM, Yuen TC, Edelson DP. Risk stratification of hospitalized patients on the wards. Chest. 2013;143(6):1758-1765.

13. Churpek MM, Yuen TC, Winslow C, et al. Multicenter development and validation of a risk stratification tool for ward patients. Am J Respir Crit Care Med. 2014;190(6):649-655.

14. Rivers E, Nguyen B, Havstad S, et al. Early goal-directed therapy in the treatment of severe sepsis and septic shock. N Engl J Med. 2001; 345(19):1368-1377.

15. Dellinger RP, Levy MM, Rhodes A, et al. Surviving sepsis campaign: international guidelines for management of severe sepsis and septic shock: 2012. Crit Care Med. 2013;41(2):580-637.

16. Vanhecke TE, Gandhi M, McCullough PA, et al. Outcomes of patients considered for, but not admitted to, the intensive care unit. Crit Care Med. 2008;36(3):812-817.
17. Metcalfe MA, Sloggett A, McPherson K. Mortality among appropriately referred patients refused admission to intensive-care units. Lancet. 1997;350(9070):7-11.

18. Churpek MM, Yuen TC, Winslow C, Hall J, Edelson DP. Differences in vital signs between elderly and nonelderly patients prior to ward cardiac arrest. Crit Care Med. 2015;43(4):816-822.

19. Kang MA, Churpek MM, Zadravecz FJ, Adhikari R, Twu NM, Edelson DP. Real-time risk prediction on the wards: a feasibility study [published April 13, 2016]. Crit Care Med. doi: 10.1097/ CCM.0000000000001716.

20. Smith GB, Prytherch DR, Schmidt PE, et al. Should age be included as a component of track and trigger systems used to identify sick adult patients? Resuscitation. 2008;78(2):109-115.

21. Duckitt RW, Buxton-Thomas R, Walker J, et al. Worthing physiological scoring system: derivation and validation of a physiological earlywarning system for medical admissions. An observational, populationbased single-centre study. Br I Anaesth. 2007;98(6):769-774.

22. Subbe CP, Kruger M, Rutherford P, Gemmel L. Validation of a modified Early Warning Score in medical admissions. QJM. 2001;94(10): 521-526.

23. Hillman K, Chen J, Cretikos M, et al. Introduction of the medical emergency team (MET) system: a cluster-randomised controlled trial. Lancet. 2005;365(9477):2091-2097. 